\title{
Análisis de la influencia de las tareas en línea en las calificaciones de los estudiantes del nivel $\mathrm{A1}^{+}$de inglés
}

Analysis of Cyber-homework influence in level A1+ English students' grades

Leonardo Efraín Cabezas. ${ }^{1}$, Martha Lucia Lara Freire. ${ }^{2}$ \& Wilson Gonzalo Rojas Yumisaca. ${ }^{3}$ Recibido: 13-09-2019 / Revisado: 07-10-209 /Aceptado: 08-11-2019/ Publicado: 05-12-2019

\begin{abstract}
DOI: $\underline{\text { https://doi.org/10.33262/cienciadigital.v3i4.2.1004 }}$

The main objective of this research was to establish to what extent the cyber-homework influenced in the grades obtained by $\mathrm{A}^{+}$students in the first, second, and third term achievement exams. The sample consisted of 40 students of the Medicine Career of the Escuela Superior Polytechnical de Chimborazo. This was a field quantitative research, which conducted a non-probabilistic sampling considering the size and characteristics of homogeneity of the population and data were directly collected. For the analysis and the tabulation of the results, the grades were obtained from the student's cyber-homework corresponding to the student's book 12 Units, as well as the grades obtained in the three achievement exams applied during each period of time in the academic period April 2018 August 2018, were considered. Data were analyzed and interpreted through the Shapiro-Wilk normality test and the research hypothesis was accepted by applying the Krusal-Wallis nonparametric statistic test which allowed to share exact information and also it facilitated to have information about extent the cyber-homework.
\end{abstract}

\footnotetext{
${ }^{1}$ Escuela Superior Politécnica de Chimborazo, Facultad de Administración de Empresas. Riobamba, Ecuador. leonardoe.cabezas@espoch.edu.ec

${ }^{2}$ Escuela Superior Politécnica de Chimborazo, Facultad de Salud Pública. Riobamba, Ecuador. mlaraf@espoch.edu.ec

${ }^{3}$ Escuela Superior Politécnica de Chimborazo, Facultad de Informática y Electrónica. Riobamba, Ecuador. wilson.rojas@espoch.edu.ec
} 
Finally, it was concluded that as the platform grades increased, students' grades in the achievement tests were also better; whereas students who did not develop the cyberhomework activities presented lower grades in the tests.

Keywords: Field Research, Quantitative Research, Virtual Platform, Statistic Tests, CyberHomework.

\section{Resumen}

El objetivo de la presente investigación fue establecer en qué medida las tareas en línea influyeron en las calificaciones obtenidas por los estudiantes del Nivel $\mathrm{A}^{+}$en los exámenes del primer, segundo y tercer parcial. La muestra estuvo conformada por 40 estudiantes de la Carrera de Medicina de la Escuela Superior Politécnica de Chimborazo. Para el presente trabajo de campo de tipo cuantitativo se aplicó un muestreo no probabilístico considerando el tamaño y las características de homogeneidad de la población y los datos se recolectaron de manera directa. Para el análisis y tabulación de resultados se consideraron las calificaciones obtenidas por los estudiantes en las tareas en línea correspondientes a las 12 Unidades que constan en el libro del estudiante, así como las notas de las tres evaluaciones parciales aplicadas durante el período académico abril-agosto 2018. Los datos fueron analizados e interpretados mediante la prueba de normalidad Shapiro-Wilk y se aceptó la hipótesis alterna de investigación aplicando la prueba no paramétrica Krusal-Wallis. Finalmente, se concluyó que los estudiantes cuyas notas en las actividades de la plataforma incrementaron, también tuvieron mejores notas en las pruebas; mientras que los estudiantes que no desarrollaron las taras en línea obtuvieron notas más bajas en las pruebas.

Palabras claves: Estudio de Campo, Investigación Cuantitativa, Plataforma Virtual, Pruebas Estadísticas, Tareas en Línea.

\section{Introducción}

En la actualidad es interés de la UNESCO de fortalecer el acompañamiento académico tanto del docente como de los estudiantes en las aulas de los centros educativos; apoyando el desarrollo de las comunidades o grupos de aprendizaje y ofreciendo acciones educativas con la finalidad que 
amplíen el tiempo y el espacio para el aprendizaje más allá de la escuela (Severín, 2013). Es así que, en el artículo titulado "Uso de Plataformas Virtuales de Aprendizaje en el desarrollo cognitivo de los estudiantes de Nivel Superior" se determina que los estándares de competencias en TICs para los docentes se encuentran enmarcados bajo tres enfoques principales: nociones básicas, profundización y generación del conocimiento, lo cual enfatiza la relevancia que tiene el uso adecuado de las tareas en línea y su influencia que tiene en las calificaciones de los exámenes parciales que obtienen los estudiantes al final de cada periodo (Damián, Celleri, Inca, \& Castillo, 2019).

En el Ecuador, la mayoría de instituciones educativas de nivel superior utilizan un texto base o material de apoyo académico para el desarrollo del proceso de enseñanza aprendizaje del idioma inglés como lengua extranjera. Estos textos y materiales tienen varios componentes que incluyen: libro digital, posters, tareas en línea, videos, audios en mp3, ejercicios de pronunciación, practica de exámenes, y proyectos de aula; los mismos que contribuyen a reforzar el rendimiento académico de los estudiantes dentro del proceso enseñanza aprendizaje (Castro, Abreus, \& Hernández, 2015).

Cabe recalcar que dentro de todos los componentes del material de estudio se encuentra las actividades de tareas en línea, también conocidas dentro del inglés como "Cyber-homework" que son actividades interactivas asignadas a los estudiantes previo a la planificación por parte del docente dentro de una plataforma virtual y en tiempo determinado para su elaboración y completamiento de las actividades. Estas actividades permiten una retroalimentación integra, completa e inmediata de todos los temas estudiados en las diferentes clases, la misma que se despliega automáticamente al término de la actividad y a la vez el docente puede almacenar la información de los resultados de cada uno de los ejercicios desarrollados por los estudiantes sin mayor cumulo de corrección y de trabajo; lo cual permite una interacción entre docente y estudiantes a pesar de las distancias de espacio y tiempo ya que estas actividades son diseñadas para ser realizadas dentro de un determinado periodo de tiempo pero a la vez apoya al proceso de enseñanza aprendizaje (Fandos, 2003).

El uso de las tareas en línea es un tema que ya ha sido investigado, por esta razón la autora Sert (2015) en su artículo titulado "Student's Attitudes Towards Cyber-Homework" manifiesta que la implementación de la tecnología a través de tareas en línea es favorable para los estudiantes, debido 
a que los motiva y a la vez contribuye significativamente en el aprendizaje del idioma inglés, lo cual permite desarrollar autoconfianza en los estudiantes al no sentirse presionados con la presencia de sus compañeros junto al miedo a equivocarse y además pueden realizar estas actividades en la comodidad y espacio que más creyeren conveniente (Sert, 2015). Lo que corrobora la relevancia que tiene el presente trabajo de investigación, considerando que en los últimos años la integración de la tecnología ha sido crucial en el ámbito educativo debido a los cambios que está sufriendo actualmente la sociedad por la introducción de la tecnología y por su incidencia significativa en todos los niveles de educación.

González (2013) en su libro titulado “Let’s work with technology” manifiesta que la tecnología, los computadores, el internet y muchas formas de interacción virtuales están motivando a que los estudiantes aprendan de diferente manera hoy en día y los docentes tengan otras opciones de impartir conocimientos; entonces por qué no tomar este precedente para incrementar a favor del proceso enseñanza-aprendizaje del idioma inglés en las diferentes actividades con la finalidad de aplicar en las diferentes habilidades del idioma para el buen desempeño educativo de los estudiantes (González, 2013).

La Escuela Superior Politécnica de Chimborazo consciente de la importancia del idioma inglés como parte de las diferentes carreras que ofrece incorpora en su pensum de estudios la aprobación de 4 niveles de inglés con una carga horaria de 8 horas semanales en las diferentes Carreras, para lo cual se implementa el uso del libro del estudiante para el desarrollo de las actividades curriculares dentro y fuera de las aulas alineados al silabo correspondiente de cada uno de los cuatro niveles de inglés. Este texto que se encuentra basado en el Marco Común Europeo de Referencia para Lenguas contiene 12 unidades de estudio y además una plataforma virtual para el desarrollo de tareas en línea de los estudiantes de nivel A1+.

Las tareas en línea son desarrolladas por los estudiantes en un tiempo determinado y asignadas por los docentes en cada una de las lecciones de estudio de las 12 unidades con la finalidad de ayudar a promover el desarrollo de las habilidades para el proceso enseñanza aprendizaje del idioma ingles tales como: comprensión auditiva, escritura, lectura, uso de gramática y vocabulario; 
las mismas que demandan de atributos que los estudiantes deben poseer, como: responsabilidad, autonomía, administración de tiempo e ingenio (Arellano, Herazo, \& Jerez, 2012).

Es importante considerar que el propósito de este estudio de campo de tipo cuantitativo fue establecer en qué medida las tareas en línea influyeron en las calificaciones durante los tres periodos parciales obtenidas por los estudiantes del Nivel $\mathrm{A}^{+}$de la Carrera de Medicina de la Escuela Superior Politécnica de Chimborazo, donde a través de los tres exámenes parciales se pudo obtener un panorama muy claro de la influencia del uso de las tareas en línea.

Finalmente, el interés principal de este estudio de investigación fue analizar la incidencia que tienen las tareas en línea asignadas a los estudiantes del Nivel $\mathrm{A}^{+}$de la Carrera de Medicina de la Escuela Superior Politécnica de Chimborazo, sobre las calificaciones obtenidas en las evaluaciones de los tres periodos parciales tomadas a los estudiantes, ya que no se dispone de esta información que se encuentra relacionada con estas variables de estudio.

\section{Plataformas Virtuales}

Son programas que junto al internet están orientados al desarrollo y uso de cursos, módulos o actividades didácticas. Las plataformas virtuales permiten una mejor comunicación entre docentes y estudiantes a través de un aprendizaje individual o colectivo (Pérez \& Saker, 2013).

Las plataformas virtuales se emplean para la educación y estas a la vez simulan las mismas condiciones de aprendizaje que se realizan en las aulas, permitiendo la interacción, las mismas que disponen de diversas vías de comunicación como chats, correos, foros de debate, videoconferencias, blogs, etc. (Ramírez, 2017).

El concepto de plataforma virtual se ha hecho muy común en el aprendizaje de idiomas; ya que, personas de cualquier parte del mundo pueden estudiar su idioma favorito sin necesidad de realizar grandes inversiones económicas y además sin la necesidad de grandes requerimientos técnicos para el funcionamiento y desarrollo de las mismas (Pérez J., 2013). 
Es importante también destacar que hay numerosas plataformas virtuales, las cuales tienen software diseñados para ayudar a los estudiantes y docentes. Permitiendo, crear, diseñar y evaluar cursos en línea de alta calidad y entornos de aprendizaje virtuales (Peralta, 2015).

\section{Tareas en línea}

Son todas las actividades ya programadas y organizadas con una estructura que están obligadas a ser resueltas. Estas tareas contribuyen al cumplimiento de actividades previamente establecidas bajo políticas de cumplimiento e interacción. Además, las tareas en línea permiten al docente mantener el control del proceso de las actividades que están desarrollando sus estudiantes; así como también, los estudiantes deben cumplir rigurosamente con los tiempos que se han establecido para completar las actividades asignadas (Delgado \& Solano, 2009).

Es decir, las tareas en línea se derivan de un rol organizativo que se ajusta a directrices que están establecidas en un programa de estudios y reflejan una de las estrategias que tiene el docente para favorecer la interacción entre estudiantes y docentes facilitando la construcción del aprendizaje (Unigarro, 2005).

\section{Ventajas y desventajas}

En una sociedad inmersa en el desarrollo tecnológico, donde la informática y la educación van de la mano, podemos ver que las tareas en líneas se han convertido en un gran apoyo tanto para el docente como para el estudiante; ya que la implementación de estas actividades junto al uso de la tecnología enriquece el proceso enseñanza aprendizaje (Jiménez \& Rubio, 2017).

Sin embargo, para que este proceso de tareas en línea cumpla con las expectativas esperadas debemos considerar ciertos factores tanto positivos como negativos, con la finalidad de tener los mejores beneficios y evitar las limitaciones para el desarrollo educativo (Arévalo, 2016). 
Tabla 1. Ventajas y desventajas de las tareas en línea

\section{TAREAS EN LINEA}

\section{ESTUDIANTES}

\section{VENTAJAS} colaborativo.

- Se adaptan a los diferentes ritmos de aprendizaje.

- Disminuyen las barreras de espacio y tiempo.

- Crean nuevos escenarios para el aprendizaje.

- Presentan más interés y motivación en los estudiantes.

- Acceso a diferentes programas, videos, audios, lecturas, chats y muchas opciones más, que favorece al aprendizaje del inglés.

- Fomentan en los estudiantes autoconfianza y disminuyen sus miedos a lo que los demás puedan decir.

- Proporcionan acceso desde cualquier parte siempre que exista conexión a internet.

\section{DESVENTAJAS}

- Mal utilizadas se convierte en distracción, especialmente en adolescentes.

- Generan cansancio visual.

- A largo plazo pueden generar dependencia.

\section{DOCENTES}

\section{VENTAJAS}

DESVENTAJAS

- Permiten llevar el control de asignar tareas, administrar contenidos, aplicar evaluaciones, y dar retroalimentación a sus estudiantes.

- Proporcionan flexibilidad sin la necesidad de tener a los estudiantes en un aula a un horario y momento específico.

- Dan seguimiento más detallado de calificaciones, tareas entregadas y participación de los estudiantes.

Fuente: Universidad Nacional Abierta y a Distancia (UNAD)

Elaborado por: Investigadores

- No adaptarse a los nuevos cambios tecnológicos por desconocimiento de cómo saber utilizar las tareas en línea.

- Requieren un mayor tiempo de dedicación. 


\section{Rendimiento académico}

La complejidad que tiene este concepto se conceptualiza como aptitud escolar, rendimiento estudiantil o desempeño académico pero las diferencias de conceptos son solo cuestiones semánticas (Heredia-Escorza, 2014). Es decir, rendimiento académico es un nivel de conocimientos demostrados en un área o asignatura junto a diferentes factores de edad, y nivel académico.

El rendimiento académico se entiende al proceso que se lleva con la finalidad de medir los logros alcanzados por los estudiantes, a través de la simple o compleja medición y evaluación (Navarro, 2003).

\section{Evaluación del rendimiento académico}

Si pretendemos conceptualizar el rendimiento académico basado en la evaluación, es necesario considerar algunos aspectos, los mimos que nos proveen pautas necesarias para medir la calidad de rendimiento. Dentro de estos aspectos no sólo se debe considerar el desempeño individual o grupal de los estudiantes sino también, la manera como influyó en el contexto educativo con la finalidad de conocer las variables que inciden en el nivel del aprendizaje.

Probablemente, una de las variables del rendimiento académico considerada por los profesores son las calificaciones que permiten calcular el desempeño de los estudiantes. Este análisis de calificaciones son predicciones del rendimiento académico y por ende el desarrollo intelectual.

Resulta importante mencionar la variable de la inteligencia, ya que no es fácilmente identificable, pero incide en el rendimiento académico del estudiante.

\section{Desarrollo del rendimiento académico}

Seguramente, basados en la situación actual del desempeño académico que tienen los estudiantes se realiza un análisis y se toman medidas emprendidas para el buen desarrollo del rendimiento académico. Estas medidas a todo nivel educativo deben estar dirigidas a la implementación de actividades académicas para evitar las carencias en la educación. 
En todos los niveles de educación se debe fomentar programas de apoyo para los estudiantes que conlleven a mejorar el rendimiento académico. Estos programas deben incluir el uso de materiales y textos de apoyo y el fortalecimiento de los aprendizajes mediante el uso de la tecnología para aportar al desarrollo académico.

\section{Objetivos}

\section{Objetivo General}

- Establecer en qué medida las tareas en línea influyen en las calificaciones obtenidas por los estudiantes del Nivel $\mathrm{A} 1^{+}$en los exámenes parciales.

\section{Objetivos Específicos}

- Examinar la relación que existe entre las calificaciones de las tareas en línea y las calificaciones de los estudiantes en los exámenes parciales.

- Identificar las unidades de estudio en las que los estudiantes presentan mayor dificultad de aprendizaje.

\section{Hipótesis}

Hipótesis nula (H0): Las tareas en línea NO influyen en las calificaciones parciales obtenidas por los estudiantes del Nivel $\mathrm{A}^{+}{ }^{+}$en los exámenes parciales.

Hipótesis alterna $\left(\mathbf{H}_{1}\right)$ : Las tareas en línea SI influyen en las calificaciones parciales obtenidas por los estudiantes del Nivel $\mathrm{A} 1^{+}$en los exámenes parciales.

\section{Métodos y Materiales}

La presente investigación es de campo, pues los datos fueron recolectados directamente de los sujetos investigados y de la realidad donde ocurrieron los hechos, sin manipular las variables de estudio (desarrollo de tareas en línea y calificaciones obtenidas por los estudiantes) o alterar las condiciones existentes (Arias, 2012). Adicionalmente, éste trabajo responde a las características de una investigación cuantitativa; puesto que se describe, comprueba y confirma la influencia de las tareas en línea en el rendimiento académico. Así mismo, la teoría se produce a partir de comparar 
resultados de los datos recolectados de la muestra y de estudios previos. Por otra parte, la investigación asegura procedimientos rigurosos y objetivos para evitar sesgos y tendencias que influyan en los resultados. Finalmente, se establecen hipótesis que posteriormente son aceptadas o rechazadas.

En cuanto a la selección de la muestra se aplicó el muestreo no probabilístico considerando el tamaño y las características de homogeneidad de la población. Y la muestra estuvo conformada por 40 estudiantes del Paralelo "E" del Nivel $\mathrm{A1}^{+}$de la Carrera de Medicina de la Escuela de la Escuela Superior Politécnica de Chimborazo. La investigación se desarrolló durante el período académico abril-agosto 2018.

Durante el período académico se aplicaron tres evaluaciones parciales. El instrumento utilizado fue el cuestionario, el mismo que constó de 30 ítems relacionados con gramática y vocabulario, 9 ítems de comprensión auditiva, 20 de lectura y una pregunta de escritura que consistió en redactar un párrafo de 150 palabras. Este instrumento de evaluación fue previamente estructurado y programado en la plataforma virtual del texto base utilizado por los estudiantes de nivel $\mathrm{A}^{+}$de inglés.

Para el análisis y tabulación de resultados se consideraron las calificaciones obtenidas por los estudiantes en las tareas en línea correspondientes a las 12 Unidades de estudio.

Las tareas en línea fueron evaluadas sobre 2 puntos y para analizar las mismas se aplicaron las siguientes pruebas estadísticas:

- Se comparó el total de notas de cada lección de la plataforma, primero se aplicó la prueba de normalidad de Shapiro-Wilk, donde se puedo observar que los datos no vienen de una distribución normal dando un $\mathrm{p}<0,05$.

- Se verificó que los datos no provienen de una distribución normal, por lo que se aplicó la prueba no paramétrica Kruskal-Wallis, para demostrar la homogeneidad de las lecciones de la plataforma, dando como resultado $\mathrm{P}<0.05$, es decir que al menos existe una mediana de los grupos que es diferente entre sí. 
- Se aplicó la prueba Tukey con la finalidad de detallar cuales fueron las notas de las lecciones que no tuvieron homogeneidad y las lecciones de la plataforma que no mostraron relación entre sí.

\section{Resultados}

Una vez aplicados los instrumentos a los estudiantes del Nivel $\mathrm{A} 1^{+}$se detallan los siguientes resultados:

Tabla 2. Notas obtenidas por los estudiantes en las tareas en línea

\begin{tabular}{|c|c|c|c|c|}
\hline \multicolumn{5}{|l|}{ HSD Tukey ${ }^{\mathrm{a}, \mathrm{b}}$} \\
\hline \multirow{2}{*}{ UNIDAD } & \multirow{2}{*}{$\mathbf{N}$} & \multicolumn{3}{|c|}{ Subconjunto para alfa $=\mathbf{0 . 0 5}$} \\
\hline & & 1 & 2 & 3 \\
\hline TOTAL UNIT 10 & 34 & 1,277530138 & & \\
\hline TOTAL UNIT 9 & 38 & 1,445541043 & 1,445541043 & \\
\hline TOTAL UNIT 11 & 35 & & 1,569929427 & 1,569929427 \\
\hline TOTAL UNIT 12 & 35 & & 1,588292487 & 1,588292487 \\
\hline TOTAL UNIT 4 & 40 & & & 1,724839858 \\
\hline TOTAL UNIT 8 & 40 & & & 1,726815376 \\
\hline TOTAL UNIT 1 & 39 & & & 1,728373621 \\
\hline TOTAL UNIT 6 & 40 & & & 1,732665322 \\
\hline TOTAL UNIT 7 & 40 & & & 1,738452717 \\
\hline TOTAL UNIT 5 & 40 & & & 1,739889164 \\
\hline TOTAL UNIT 3 & 40 & & & 1,771074908 \\
\hline TOTAL UNIT 2 & 39 & & & 1,775219827 \\
\hline Sig. & & 0,252 & 0,509 & 0,056 \\
\hline
\end{tabular}

Se visualizan las medias para los grupos en los subconjuntos homogéneos.

a. Utiliza el tamaño de la muestra de la media armónica $=38,198$.

b. Los tamaños de grupo no son iguales. Se utiliza la media armónica de los tamaños de grupo. Los niveles de error de tipo I no están garantizados.

\section{Fuente: Plataforma Virtual Elaborado por: Investigadores}

Observando la tabla podemos ver tres grupos muy definidos, de los cuales el primer grupo está conformado por las unidades más homogéneas entre sí unidad 9 y 10. Segundo grupo por las unidades $9,11,12$. Finalmente, el tercer grupo por las unidades $1,2,3,4,5,6,7,8,11$ y 12. 


\section{Análisis estadístico de las notas parciales de la plataforma virtual del semestre.}

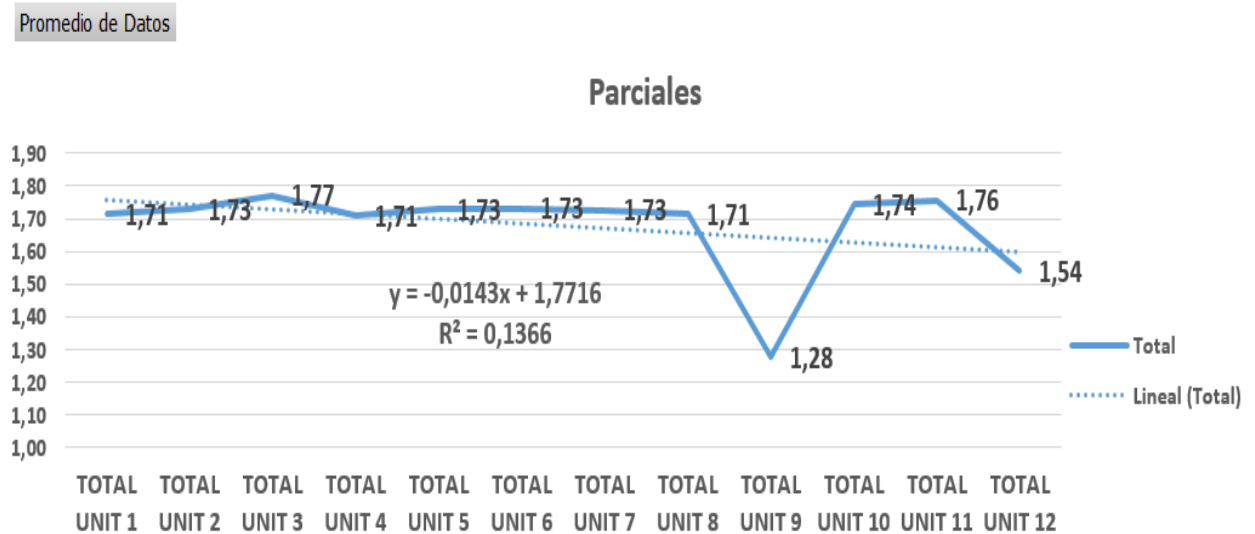

Categoria

Figura 1. Diagrama de dispersión

Fuente: Software estadístico R

Elaborado por: Investigadores

Para el cálculo de regresión lineal simple se utilizará la siguiente formula:

$$
\mathrm{y}=\mathrm{B}_{0}+\mathrm{B}_{1} \mathrm{X}_{\mathrm{t}}
$$

Para el análisis de los resultados entre las notas de la plataforma de manera general y de los datos de los parciales de los estudiantes, se realiza un análisis de regresión para observar si existe una dependencia de las notas de la plataforma para obtener una buena nota en los exámenes parciales, dando como resultado una ecuación lineal de:

$$
y=0,8579+0,4565 x
$$

Donde podemos observar que existe que $\mathrm{B}_{\mathrm{o}}$ es el cambio medio que produce las notas de los exámenes parciales por cada cambio que hace las notas de la plataforma.

Es decir que al trazar una pendiente en el gráfico de dispersión podemos manifestar que por cada 0,45 de promedio en las calificaciones de la plataforma existe un incremento en una unidad, en otras palabras, por cada incremento en las notas de la plataforma, va existir un mayor incremento en las notas de los parciales. 


\section{Discusión}

La investigadora Ceyda Sert realizó un estudio en una institución educativa privada de nivel secundario en Antalya, Turquía, con la finalidad de explorar el rol de las nuevas herramientas de aprendizaje en línea y el efecto de las tareas en línea sobre el éxito en el aprendizaje del idioma inglés. Este estudio se realizó con una muestra de 201 estudiantes pertenecientes a los grados $5^{\text {to }}$ $6^{\text {to }}$ y $7^{\text {mo }}$, a quienes se les asignaron tareas en línea. Los resultados obtenidos en dicho estudio revelaron que el 39\% de estudiantes mostraron interés por el cyber-homework y tuvieron éxito en sus exámenes.

Así mismo, en la publicación efectuada por Ji Hye Shin y Peggy Alberts, en la que se examinó la efectividad del Sistema de Aprendizaje de Tareas en Casa (SATC) se demostró que no existieron diferencias estadísticas significativas en el rendimiento del examen entre los grupos experimental y de control. El (SATC) puede ser considerado un suplemento útil, pero no un remplazo para las tutorías privadas de secundaria (Albers, 2015).

La presente investigación se trata del análisis de la influencia de las tareas en línea en las calificaciones de los estudiantes del nivel $\mathrm{A}^{+}$de inglés. Y tiene como propósito establecer en qué medida las tareas en línea influyen en las calificaciones parciales obtenidas por los estudiantes del Nivel $\mathrm{A} 1^{+}$en los exámenes parciales.

Este estudio se desarrolló mediante el uso de una plataforma virtual que le permitió al estudiante descargar textos digitales, audios, videos, así como también prácticas de escritura, evaluaciones, y ejercicios de pronunciación.

De igual manera, el estudiante tuvo la oportunidad de realizar las actividades programadas por el docente mediante la utilización de las tareas en línea, siendo necesario dividir el número de unidades de la plataforma de una manera adecuada con la finalidad de que coincidan con las evaluaciones parciales que debieron ser realizadas por los estudiantes en el semestre; en este caso tres parciales de 4 unidades cada uno, que constaron en el calendario académico. Los temas 
utilizados en los ejercicios desarrollados por parte de los estudiantes fueron: ejercicios de lectura, vocabulario, gramática, diálogos y audio.

Khon (1993) manifiesta que el motivador esencial para los estudiantes es el temor a una mala calificación, esto se comprobó en los resultados de las tareas en línea de las 12 unidades, donde las 8 primeras fueron obligatorias y las 4 últimas optativas. La participación de los estudiantes en las actividades obligatorias fue activa; no así, en las optativas (Farias, 2010). Basado en la afirmación de Khon se pudo evidenciar que los estudiantes de la Carrera de Medicina mostraron interés por realizar las actividades asignadas en la plataforma al recibir una calificación.

Igualmente, los resultados demostraron tres grupos determinados por los resultados de las unidades más homogéneas.

En el primer grupo de las unidades 9 y 10 se pudo determinar que el número de participantes fue inferior a los otros dos grupos y que las calificaciones fueron inferiores como se muestra en la Tabla 2.

El segundo grupo conformado por las unidades 9, 11, y 12, muestra un mejoramiento en la calificación; a pesar de que, el número de participantes es inferior al grupo tres.

Finalmente, como un aspecto muy positivo el tercer grupo que constituyen las unidades 1, 2, 3, 4, 5, 6, 7, 8, 11 y 12 tiene un mayor número de participantes y una calificación más alta en las actividades designadas. Estos resultados revelaron que si existe una dependencia de las notas de la plataforma para obtener una buena nota en los exámenes parciales.

\section{Conclusiones}

De acuerdo a los datos encontrados e interpretados en este trabajo de investigación, se acepta la hipótesis alterna que indica que las tareas en línea sí influyen en las calificaciones obtenidas por los estudiantes del Nivel $\mathrm{A}^{+}{ }^{+}$de la Carrera de Medicina de la Escuela Superior Politécnica de Chimborazo como lo indican los datos estadísticos de las Tablas 3 y 4. Sin embargo, se debe recalcar que existió un mayor incremento en las calificaciones que correspondieron a las actividades realizadas de manera obligatoria. 
Este estudio también permitió identificar que las ventajas del uso de las tareas en línea tanto para los docentes como para los estudiantes fueron superiores a las desventajas; en lo concerniente a los docentes, se comprobó que el uso de la plataforma facilitó el control y retroalimentación de las actividades evaluadas, en menor tiempo. En cuanto a los estudiantes, se evidenció el fortalecimiento del aprendizaje autónomo, disminuyendo así las barreras de espacio y tiempo, fomentando la autoconfianza y creando nuevos escenarios para el aprendizaje.

Como desventajas se identificaron la falta de conocimiento y adaptación en los docentes a los nuevos cambios tecnológicos, requiriendo un mayor tiempo de dedicación para el uso efectivo de las tareas en línea. Mientras que para los estudiantes la desventaja fue el limitado acceso de Internet para realizar las tareas asignadas.

Frente a la evidencia recaudada en la Tabla 2, se concluye que la unidad 10 es la que presenta mayor dificultad de aprendizaje; ya que esta unidad fue asignada de forma optativa siendo el 15\% de estudiantes quienes no completaron la tarea.

En relación a lo antes expuesto, es importante recalcar que las tareas en línea constituyen una herramienta de mucha utilidad para los docentes del idioma inglés como Lengua Extranjera y que su adecuada implementación fortalece el proceso de enseñanza-aprendizaje y motiva la participación activa de los estudiantes fuera del aula de clase mejorando así su rendimiento académico.

En conclusión, por cada incremento en las notas de la plataforma existió un mayor incremento en las notas de los exámenes parciales de los estudiantes del Paralelo "E” del Nivel A1+.

\section{Referencias Bibliográficas}

Arboleda, M. (18 de diciembre de 2017). Estadísticas de redes sociales en Ecuador 2017. Obtenido de BRANDEC: http://www.hablemosdemarcas.com/estadisticas-de-redessociales-en-ecuador-2017/

Basantes, A. (2017). Formación universitaria. Obtenido de Los Dispositivos Móviles en el Proceso de Aprendizaje de la Facultad de Educación Ciencia y Tecnología de la Universidad Técnica del Norte de Ecuador: 
https://scielo.conicyt.cl/scielo.php?script=sci_abstract\&pid=S071850062017000200009\&lng=es\&nrm=iso

Caldero, R. (15 de mayo de 2014). Obtenido de El uso del teléfono móvil y la aplicación WhatsApp para el aprendizaje de verbos frasales ingleses en alumnos de nivel intermedio. España: Universidad Internacional de la Rioja.: https://reunir.unir.net/bitstream/handle/123456789/2639/caldero\%20gene.pdf?sequence= 1\&isAllowed=y

Calero, M. (2014). El discurso del WhatsApp. Obtenido de https://s3.amazonaws.com/academia.edu.documents/35650265/Calero._Oralia_17.pdf?res ponse-contentdisposition=inline\%3B\%20filename\%3DEl_discurso_del_WhatsApp_entre_el_Messen.p df\&X-Amz-Algorithm=AWS4-HMAC-SHA256\&X-AmzCredential=AKIAIWOWYYGZ2Y53UL3A\%2F201911

Lebrón, A. (3 de Julio de 2009). LA ENSEÑANZA DE VOCABULARIO EN EL AULA DE. Obtenido de Temas para la Educación: https://www.feandalucia.ccoo.es/andalucia/docu/p5sd5037.pdf

Lenin Lara, M. A. (2017). Uso didáctico de WhatsApp para desarrollar la participación de los estudiantes en él. Revista Dilemas Contemporáneos: Educación, Política y Valores., 19. Obtenido de http://files.dilemascontemporaneoseducacionpoliticayvalores.com/200003715c4c06c5bf2/18.1.17\%20Uso\%20did\%C3\%A1ctico\%20de\%20WhatsApp\%20para\%20des arrollar\%20la...pdf

Moll, S. (19 de enero de 2014). ¿En qué consiste la actividad de aprendizaje cooperativo Emojiónate? Obtenido de https://justificaturespuesta.com/actividad-de-aprendizajecooperativo-emojionate/

Prado, J. (2015). ESTRATEGIAS Y ACTIVIDADES PARA EL USO DEL DICCIONARIO EN EL AULA. Obtenido de file:///C:/Users/NEGRO/Downloads/4655Texto\%20del\%20art\%C3\%ADculo-6955-1-10-20121207.pdf

RAMÍREZ, M. A. (2017). APRENDIZAJE COLABORATIVO A TRAVÉS DE WHATSAPP Y FACEBOOK. Congreso Nacional de Investigación educativa, 9.

Recursos Educativos. (2016). ¿EL MÓVIL EN EL AULA? IDEAS, VENTAJAS, RETOS Y POSIBILIDADES. Obtenido de Inspira TICs: https://www.inspiratics.org/es/recursoseducativos/el-movil-en-el-aula-ideas-ventajas-retos-y-posibilidades 
Rubio, J. (7 de junio de 2015). El fenómeno WhatsApp en el contexto de la comunicación personal: una aproximación a través de los jóvenes universitarios. Obtenido de ICONO 14: https://www.icono14.net/ojs/index.php/icono14/article/view/818

Telégrafo. (29 de Julio de 2017). WhatsApp alcanza los 1.000 millones de usuarios a diario. pág. 1. Obtenido de https://www.eltelegrafo.com.ec/noticias/tecnologia/1/whatsapp-alcanzalos-1-000-millones-de-usuarios-a-diario

UNIVERSIA. (26 de Julio de 2016). WhatsApp para fines educativos: recomendaciones para docentes y estudiantes. Obtenido de https://noticias.universia.pr/educacion/noticia/2016/07/26/1142129/whatsapp-fineseducativos-recomendaciones-docentes-estudiantes.html

Vázquez, F. (5 de octubre de 2016). WhatsApp como herramienta de Marketing Digital. Obtenido de https://br.escueladenegociosydireccion.com/business/whatsapp-y-marketingdigital/

WIKY ESTUDIANTES, ORG. (enero de 21 de 2014). ¿POR QUÉ ES IMPORTANTE EL VOCABULARIO EN UNA LENGUA EXTRANJERA? Obtenido de https://www.wikiestudiantes.org/por-que-es-importante-el-vocabulario-en-una-lenguaextranjera/ 


\section{PARA CITAR EL ARTÍCULO INDEXADO.}

Cabezas, L., Lara Freire, M., \& Rojas Yumisaca, W. (2019). Análisis de la influencia de las tareas en línea en las calificaciones de los estudiantes del nivel A1+ de inglés. Ciencia Digital, 3(4.2), 55 -72. https://doi.org/10.33262/cienciadigital.v3i4.2.1004

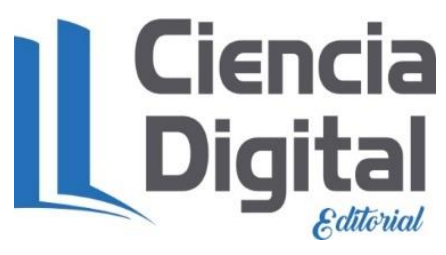

El artículo que se publica es de exclusiva responsabilidad de los autores y no necesariamente reflejan el pensamiento de la Revista Ciencia Digital.

El artículo queda en propiedad de la revista y, por tanto, su publicación parcial y/o total en otro medio tiene que ser autorizado por el director de la Revista Ciencia Digital.
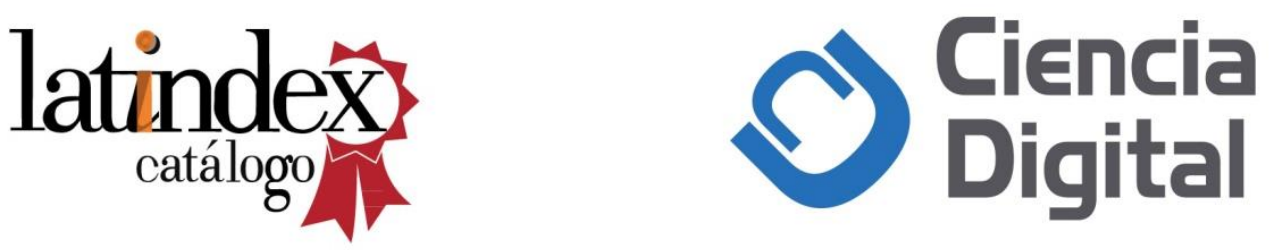